\title{
1
}

\section{Mobile Peer-to-Peer Networks: An Introduction to the Tutorial Guide}

Frank H. P. Fitzek

Aalborg University, ff@es.aau.dk

Hassan Charaf

Budapest University of Technology and Economics, Hassan@aut.bme.hu

\subsection{Introduction and Motivation}

The main aim of this chapter is to provide the reader with a good introduction to mobile peer-to-peer networks and to demonstrate the motivation behind mobile peer-to-peer network development - the basic purpose of this book. Mobile peer-to-peer networks rely on direct communication among mobile devices of any kind. Even though today's mobile communication architectures are mainly centralized and therefore based on preinstalled infrastructure, this book advocates mobile peer to peer (P2P), breaking ground for new business models and appealing services. To realize these novel communication architectures, a tutorial guide for different wireless technologies is presented in this book. Roughly speaking, this book deals with the potential to build mobile networks without or with the help of cellular networks, taking advantage of the short-range technologies Bluetooth and WiFi (IEEE 802.11) to communicate among mobile devices.

Peer-to-peer networks have raised a great deal of attention in the last decade. These peer-to-peer networks started in the wired domain. First implementations targeted file-sharing services and distributed computing. The main idea was to use distributed storage and computational capacity instead of one centralized server. Such an approach was less vulnerable to denial of service attacks and made the networks more robust. As most peer-to-peer networks started with music file sharing, they were often regarded as

Mobile Peer to Peer $(P 2 P)$ Edited by Frank H. P. Fitzek and Hassan Charaf

(C) 2009 John Wiley \& Sons, Ltd 
pirate networks. The new architecture was a huge threat to the music industry, and to this day several network and service operators are still frightened by the term 'peer to peer'.

On the other hand, peer-to-peer networks have shown their potential and advantages over centralized approaches. Inspired by the fixed domain, peer-to-peer networks are now penetrating the wireless and mobile domain. The goal of this chapter is also to show the potential of mobile peer-to-peer networks as an extension to existing infrastructure approaches. Therefore, network and service operators should see the mobile peer-to-peer networks not as a threat but as huge potential to make their existing networks and services more appealing, more robust, and less resource intensive. In other words, the peer-to-peer networks define the infrastructure for different types of protocol, applications, and services.

To the best of our knowledge, the first mobile peer-to-peer service was a dating client. The main idea was that people with the same interest in meeting other people could specify their interests and needs by using a mobile application, which was realized on a Symbian platform. Using Bluetooth, the mobile application looked out for matching partners having mainly the same interests and needs. This example gives a first idea of how mobile peer-to-peer application might look. However, as explained later in this chapter, new ideas of social mobile networking and cooperative networking are being implemented in the field.

In the following, the evolution of mobile and fixed communication systems is explained to provide a common understanding for the reader.

Today's dominant mobile communication architectures can be summarized as centralized, which means that mobile devices, as soon as they are switched on, will search for any base station and/or access point, as they will assume that this is the main service access point. Once they are connected, they will search for content that is mostly stored in a centralized manner. The base stations cover a larger area by spanning multiple cells over it, one base station covering one or multiple cells by sectorized antenna patterns. But where does this centralized architecture have its origin? There are two main factors. When the first mobile devices were created for voice services, they could already communicate between each other (just like walkie-talkies), but there was also the need to communicate with people connected through fixed networks, which had already been established decades before. So bridges from the mobile world to the wired world were needed - the origin of the base stations. The second factor is that wireless communication is mainly based on radio propagation, which limits the range of mobile-to-mobile communication with respect to power constraints. Therefore, the fixed networks with their bridging capabilities allowed two mobile devices to be connected over long distances, the fixed networks acting like virtual tunnels (see Figure 1.5).

This centralized architecture has been used extensively for second- and third-generation mobile communication systems, referred to as $2 \mathrm{G}$ and $3 \mathrm{G}$. Mobile devices focus on base stations only, as these are the main source of any information. The $2 \mathrm{G}$ mobile services were mainly voice services and text messaging. With $3 \mathrm{G}$ networks, data communication has been boosted. While $2 \mathrm{G}$ networks allowed data communication at small data rates, $3 \mathrm{G}$ communication systems target data rates to support broadband Internet access. However, the centralized approach also has its drawbacks.

Following Moore's law, the computational power will double every two years, and the increased computational power will be transferred to new services for the mobile device, making it more attractive to the customer. These services include digital cameras, music 


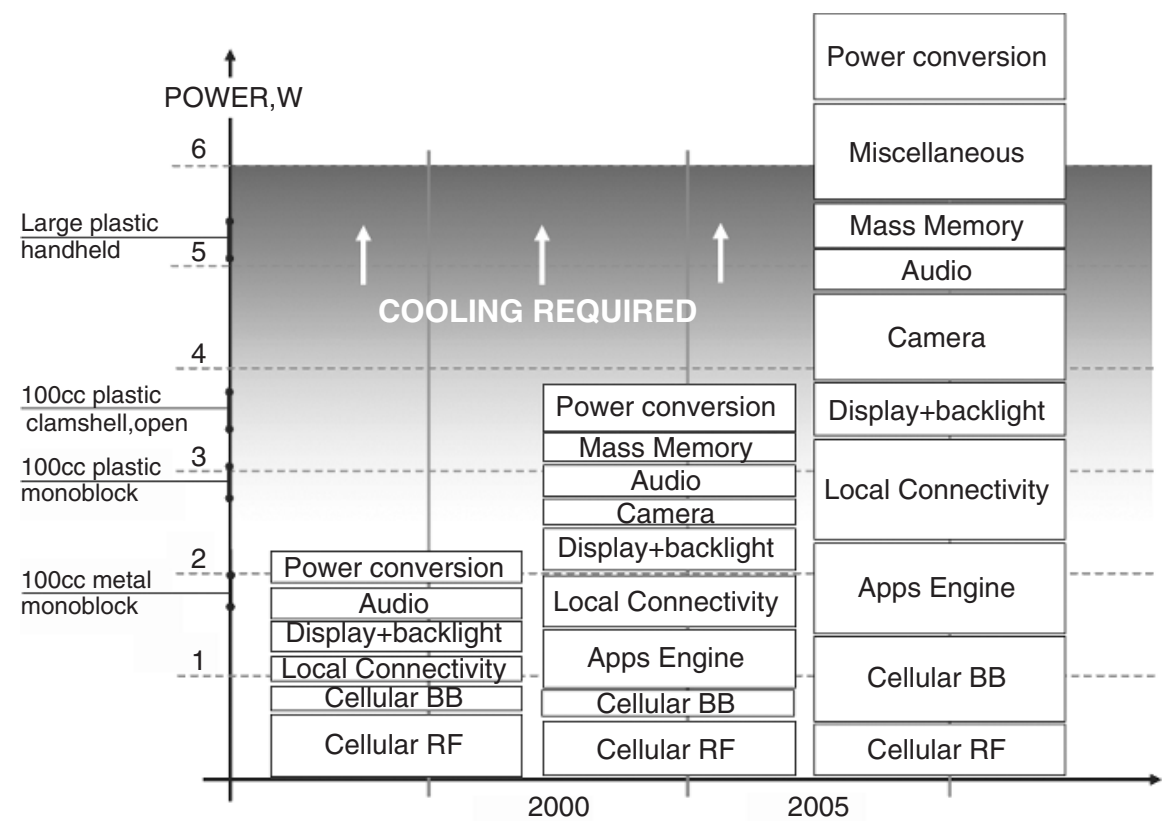

Figure 1.1 Power consumption over the last decade, with the increasing number of services on mobile devices. Reproduced by Permission of (c) 2009 Nokia. All rights reserved

players, games, and, of course, wireless technology. The latter is becoming dramatically more complex for the upcoming fourth generation $(4 \mathrm{G})$. On the other hand, we have the battery of the mobile device, which has to support the more complex services. Figure 1.1 shows the power consumption over the last decade. An increasing number of services and hardware will require more power from the battery. Unfortunately, battery capacity is not keeping pace with the development of computational power. Battery capacity has been almost doubled over the last decade. Thus, mobile users will find that their operational time, also referred to as standby time, may decrease in the future. This trend was already noticeable on transition from $2 \mathrm{G}$ to $3 \mathrm{G}$, where $3 \mathrm{G}$ mobile devices had half the operational time of $2 \mathrm{G}$ devices.

Another paradigm shift is where services are generated for the user. So far, mobile devices have been used for voice communication between people who know each other, or for data connection to the Internet. With the high-capability mobile devices (we call them smart devices or smartphones) on the market, mobile devices are not only consuming content, they are also capable of producing mobile content and to store it on their large memory. Thus, in the future, the services we are looking for might not be stored in the backbone or overlay networks. They might be right next to us on a mobile device in a range of some metres.

After understanding the origin of centralized mobile networks, we will now explain some of the advantages of moving to a peer-to-peer architecture. As explained throughout the book, mobile peer-to-peer networks have the potential to overcome the aforementioned problems. As shown in Figure 1.2, 'mobile peer to peer (P2P)' encompasses different 


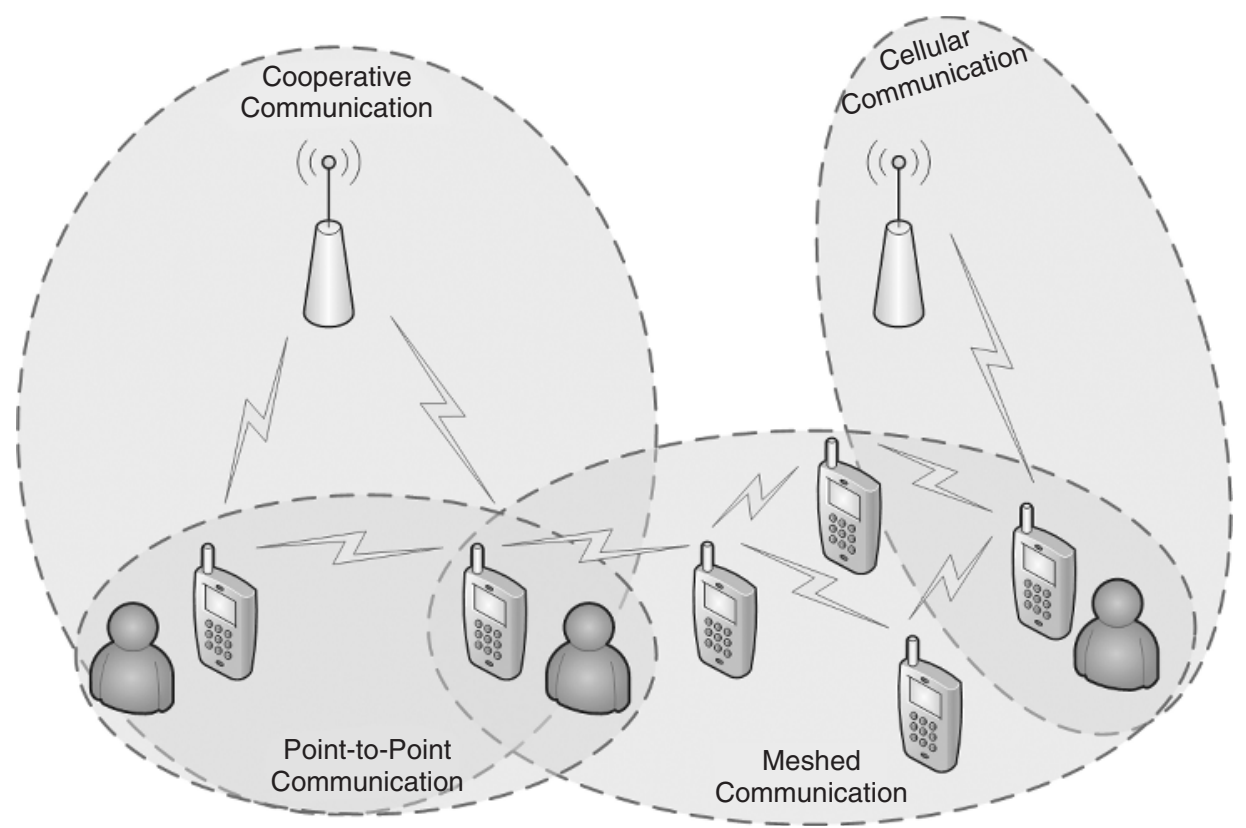

Figure 1.2 Overall architecture

architectures such as point to point, meshed networks, and cooperative networks. Network operators are often scared away by the term 'peer to peer'. This is mainly motivated by their existing business models and the fear of losing ground to peer-to-peer technology. However, network operators should regard peer-to-peer technology as a great opportunity to establish new services and break new ground to make money. Interestingly, in Africa, where fixed networks are either not established or only insufficiently so, wireless networking is growing dramatically. In dense areas, the centralized approach is used again, but, in less dense areas, new architecture forms, such as meshed networks, are considered for deployment.

\subsection{Wireless Technologies}

There are many types of wireless technology deployed on mobile handsets. Here we provide a short description of the wireless technologies used within this book. Enough information about the basic concepts should be provided for the reader to understand the following chapters.

In Figure 1.3 the supported data rates versus communication range are given for different wireless technologies. The figure shows $2 \mathrm{G}$ technologies such as GSM CSD/GPRS and $3 \mathrm{G}$ technologies such as HSDPA. Also, future technologies, referred to as $3.5 \mathrm{G}$, are named, such as worldwide interoperability for microwave access (WiMAX) and long-term evolution (LTE). Furthermore, wireless local area network (WLAN) or WiFi technologies are presented, namely different versions of IEEE802.11 and Bluetooth. In the following subsections, these technologies are outlined briefly. 


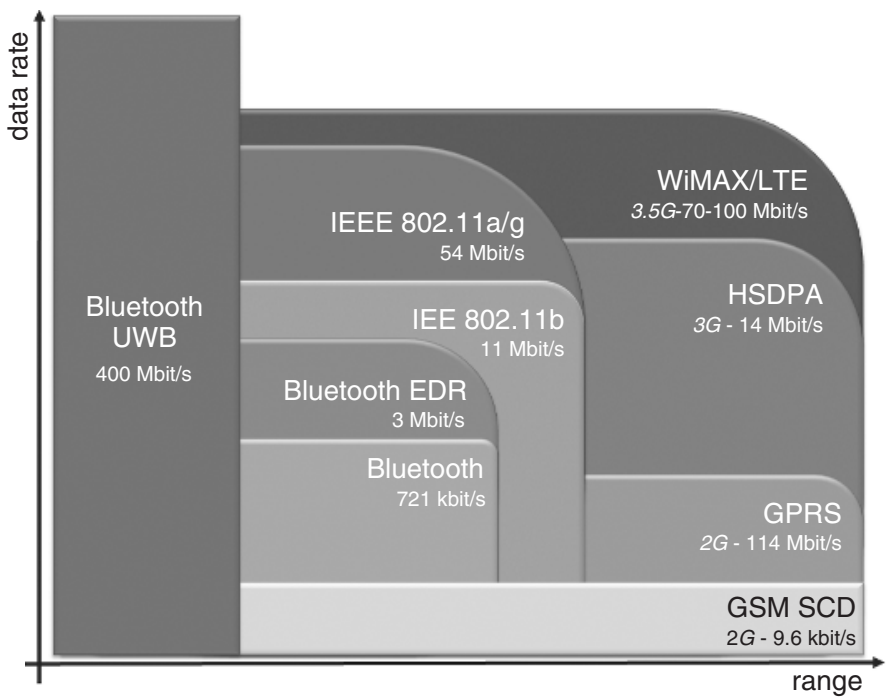

Figure 1.3 Wireless technologies and the supported data rate and range

\subsubsection{Short-range Technologies}

Here, the basic concepts of Bluetooth and WiFi (IEEE802.11) are laid out. We will mainly look into these technologies because they can be found on many mobile devices already. While Bluetooth is by far the most widely used on mobile devices today, IEEE802.11 is breaking some ground on the more advanced mobile devices, referred to as smartphones. We will not look into ZigBee, as there are only a small number of devices supporting it, and neither will we look into IRDA, as it requires line of sight.

\subsubsection{Bluetooth}

Bluetooth is a radio technology that operates in the $2.4 \mathrm{GHz}$ band. It is often referred to as short-range communication, as the range of communication is relatively small compared with cellular systems. The communication range is determined by the power class of the Bluetooth module. There exist three different Bluetooth classes, namely class 1 , class 2 , and class 3. Class 1 devices can have communication ranges of $100 \mathrm{~m}$ and even more, while class 2 and class 3 devices are limited to $10 \mathrm{~m}$ or less than $1 \mathrm{~m}$ respectively. Most mobile devices are class 2, while Bluetooth access points are class 1 . Bluetooth systems are composed of a radio/baseband part and a software stack.

Originally, Bluetooth was intended as a cable replacement. The first applications of Bluetooth were described as connecting PCs and laptops to printers. Bluetooth has since shown a much wider range of applications. It eases the process of connecting cordless peripherals such as headsets or GPS modules.

Bluetooth offers different communication profiles to define which service can be supported at a given time. Voice profiles are used for headsets connected to a mobile phone, while the LAN profile is used for data communication between two peers for IP traffic. 
In the early time of Bluetooth, a device could only support one of the profiles, while nowadays most if not all devices support multiprofile. This is needed, for example, in the case of a mobile phone connected to a headset and a PDA at the same time. Choosing the phone number on the PDA, setting up the call over the phone, and talking over the headset is only possible with multiprofile Bluetooth chipsets.

Bluetooth chipsets were advertized initially as a technology with a bill of materials (BOM) cost of \$US 5. Unfortunately, today the chipsets cost around \$US 30 if bought in small numbers. Even with a larger number, the \$US 5 threshold cannot be achieved.

Bluetooth communication takes places between one master device and at least one but a maximum of seven active slave devices. All slave devices are connected to the master device only. The numbers listed here refer to active devices. As the master is able to park a device, the master could theoretically be connected to more devices, but the number of active ongoing communication partners is restricted to seven active devices. As a result of this architecture, slaves cannot communicate directly with each other and are dependent on the master to relay information. Note that only point-to-point communication is possible, and no broadcast or multicast is possible for the slaves. Some Bluetooth implementations allow the master to broadcast information to all slaves at the same time.

To discover other Bluetooth devices in the vicinity, each device can start service discovery. The service discovery will search for other devices and classify them into mobile phones, PCs, headsets, etc. Once these devices are found, they can be paired - a procedure by which devices are approved as communication partners. In the case of a large number of Bluetooth devices, the discovery process can take quite a long time. With more than 10 devices around, it can take minutes to discover all neighbouring devices.

One Bluetooth device has the ability to support three synchronous or eight asynchronous communication channels. The synchronous channels are used for voice services mostly, while the asynchronous channels are for data communication. As we use mostly data connections in this book, we will describe these in a little bit more detail.

Because Bluetooth operates in the $2.4 \mathrm{GHz}$ bandwidth, it employs frequency hopping to make the entire communication less error prone in the presence of other technologies using this open ISM (industrial, scientific, and medical) band. Medium access is organized in a time division multiple access (TDMA) fashion, where the channel is split into $0.625 \mathrm{~ms}$ slots. Whenever one device is sending information to another device, the receipt of this information needs to be acknowledged in the next slot. In the case of unbalanced data transfer, such as the transmission of a photo from one device to another, one device is sending the data and the other one is just sending acknowledgements. The acknowledgements also occupy a full slot, which is not very efficient. To increase the efficiency, three or five slots can be bundled by one device and are acknowledged only by one slot. Furthermore, Bluetooth has the option to protect the data by forward error correction (FEC) information. Those with FEC are referred to as DM packets, and those without are referred to as DH packets. Each of these packet types can use one, three, or five slots, ending up with six different packet types, namely DM1, DH1, DM3, DH3, DM5, and DH5. Whether to use DM or DH packets depends on the signal quality. DH packets offer more capacity than DM packets, but it may be that these packets will be retransmitted more often as they are lost and therefore not acknowledged. DM packets have been used in cases where the wireless medium was highly error prone. Recent findings show that $\mathrm{DH}$ packets are more or less as robust as DM packets. This is due to novel achievements 
with hardware, more precisely with transmitter/receiver sensitivity. On this basis, new Bluetooth technologies such as ULE and UWB will not use DM packets at all.

Standard Bluetooth can achieve data rates of $721 \mathrm{kbit} / \mathrm{s}$. Using the enhanced data rate (EDR), data rates of up to $3 \mathrm{Mbit} / \mathrm{s}$ are available, as shown in Figure 1.3.

Programming examples for Bluetooth are explained in Chapter 4 and used in some of the following cases.

\subsubsection{IEEE 802.11}

IEEE802.11 describes a whole product family. The 802.11 family is based on one medium access protocol and different physical-layer implementations. Initially, 802.11 had three forms of realization at the physical layer, namely direct sequence spreading (DS), frequency hopping $(\mathrm{FH})$, and infrared (IR). As IR was limited to line of sight and FH at that point in time was more complex to realize than DS, all chipsets used DS. FH and DS were not intended to realize medium access but to improve multipath interference. The first DS realizations offered data rates of up to 1 or $2 \mathrm{Mbit} / \mathrm{s}$ working in the $2.4 \mathrm{GHz}$ frequency band. Shortly after that, $802.11 \mathrm{~b}$ was introduced, offering data rates of up to $11 \mathrm{Mbit} / \mathrm{s}$. Three fully orthogonal channels can be used to avoid interference with neighbours. As the $2.4 \mathrm{GHz}$ frequency band started to become crowded, IEEE802.11a was introduced, working in the $5 \mathrm{GHz}$ band. More orthogonal channels are now available (depending on the region, up to 12 channels for indoor use), and data rates of up to $54 \mathrm{Mbit} / \mathrm{s}$ are supported. Besides the change in frequency band from 2.4 to $5 \mathrm{GHz}, 802.11$ a uses OFDM for higher spectral efficiency. As OFDM technology demonstrated some benefits over the DS technique, IEEE802.11g was introduced, also using OFDM in the $2.4 \mathrm{GHz}$ band. Seeing as both $802.11 \mathrm{~b}$ and $802.11 \mathrm{~g}$ work in the same frequency band and have the same MAC protocol, these two technologies are nowadays often implemented on the same chipset.

For both $802.11 \mathrm{a}$ and $802.11 \mathrm{~g}$, the maximum data rate of $54 \mathrm{Mbit} / \mathrm{s}$ will only be achieved if the communicating stations have a high signal-to-noise ratio (SNR) on their communication link. Loosely speaking, the SNR decreases with increasing distance between the stations. Other factors such as shadowing, multipath, interference, etc., also play a role, but, to keep things simple, we refer to the distance. Depending on the SNR values, the stations will adapt their modulation and coding scheme. Therefore, the data rate decreases with decreasing SNR, which in turn depends on the distance between the stations.

To help the reader understand what follows, we would like to emphasize the medium access control (MAC) of IEEE802.11 in the distributed coordinating function (DCF). The MAC is based on carrier sense multiple access with collision avoidance (CSMA/CA). This means that all stations sense the medium in order to ascertain whether the medium is already busy. If this is the case, the sensing station will not send at all, to avoid collisions. Collisions occur if more than one station is using the wireless medium, and the sender will receive multiple overlay signals which it cannot receive successfully. Whenever the medium is sensed as free, the station prepares to send on the medium. As there are possibly other stations also waiting to use the medium, each station has to wait for a certain time before transmitting anything. These waiting times are different from station to station. The station with the smallest waiting time will send first. This means that the medium is busy again, and the other stations will freeze at this point in time, waiting for the next free period to come. When a station has sent a packet, it 
will wait for an acknowledgement from the counterpart communication device. If there is no acknowledgement, then the station will assume that the previous transmission has undergone a collision with at least one other station. Such collisions are still possible, as two or more stations could have had the same random timer. In this case the waiting time for the next packet will be doubled to produce more time diversity. In contrast to Bluetooth, the channel is not equally slotted. A station will occupy the medium as long as it takes to transmit the packet. This time depends on the length of the packet and the supported data rate. In addition to the sending time, the time for acknowledgement needs to be taken into consideration. Between the sending and the acknowledgement there is a small time when the medium is not being used. To prevent other stations from starting to transmit in these pause intervals, 802.11 has introduced different timers. The station responsible for sending the acknowledgement will access the medium immediately after receipt of the packet. Other stations will need to wait a longer time, and, when this timer expires, the acknowledgement will already be on its way, stopping other stations accessing the medium.

As collisions reduce the efficiency of the communication system, in 802.11, readyto-send (RTS) and clear-to-send (CTS) messages are used to avoid potential collisions. RTS messages are sent out by the sending station to ask the receiver whether it is currently busy with other transmissions of which the sending station is unaware. When the receiving station is ready, it will send the CTS message. After successful receipt of the CTS, the sending station starts to convey its message. The neighbouring stations are also informed by the RTS and CTS messages that the medium will be busy for some time. At the very least, no collisions should occur with those stations that have received either the RTS or the CTS message.

In IEEE802.11, unicast and broadcast messages can be used. Unicast is the communication between two stations, while broadcast describes the communication originated by one station and received by multiple stations. The unicast data rate is determined by the SNR between the communication partners. In broadcast, the data rates should be set according to the link with the weakest signal. Most 802.11 implementations use the lowest possible data rate whenever broadcast messages are used. Only a few chipsets allow the data rate to be set in the case of broadcast. A combination of unicast and multicast transmission is the opportunistic listening approach. Here, two stations are in communication in normal unicast mode, and the neighbouring devices are overhearing the communication. This approach has some advantages over broadcast in that at least one acknowledgement will be received by the sender.

In Chapter 5, WiFi programming examples for the Symbian OS are explained. Moreover, additional information for WiFi technology is given.

\subsubsection{2G/3G Cellular Communication}

Cellular data communication was introduced with the GSM standard as the second generation of cellular systems. Cellular systems cover larger areas. In the case of $2 \mathrm{G}$, nearly the whole of Europe is covered, apart from some very small areas. The first data rate supported, in circuit switch data (CSD) mode, was $9.6 \mathrm{kbit} / \mathrm{s}$. This was increased slightly by high-speed circuit switch data (HSCSD), using a new modulation technique for mobiles placed near the base station. With the introduction of GPRS, the data rate was improved to $114 \mathrm{kbit} / \mathrm{s}$. Both $2 \mathrm{G}$ technologies were based on TDMA technology. With the introduction 
of the third generation of cellular communication, the data rate was increased to $384 \mathrm{kbit} / \mathrm{s}$ with UMTS, and later high-speed downlink packet access (HSDPA) offered 1.5 Mbit/s on mobile devices (PCMCIA cards for laptops may achieve higher data rates). In contrast to $2 \mathrm{G}$ technologies, $3 \mathrm{G}$ is based on WCDMA instead of pure TDMA. The data rates of some $2 \mathrm{G}$ and $3 \mathrm{G}$ technologies are given in Figure 1.3. For a full explanation of these technologies, see reference [1]. Chapter 6 gives some programming examples using the Symbian OS for cellular communication.

\subsubsection{Future Wireless Trends}

In the future, the data rates of short-range and cellular systems will be improved. The family of cellular systems will be extended by WiMAX and LTE. These technologies will be available first at some hot spots, such as cities, not covering the same area as $2 \mathrm{G}$ and $3 \mathrm{G}$, but will increase their coverage with time. The data rate of WiMAX is $72 \mathrm{Mbit} / \mathrm{s}$ per cell, and the data rate of LTE is $100 \mathrm{Mbit} / \mathrm{s}$ per cell.

Improvements are also expected for the short-range technologies. As one example, we will look into the evolution of Bluetooth technology. As shown in Figure 1.4, Bluetooth, in its current implementation in version 2.0, will develop in two directions. The first direction will target higher data rates. This will be achieved by ultra-wideband (UWB) Bluetooth, with more than $400 \mathrm{Mbit} / \mathrm{s}$ between two peers over a very short range. The other direction will focus more on connectivity than on data rate. As already explained, the service discovery may take some time if multiple peers are involved. With ultra-low-power (ULP) Bluetooth, this problem is tackled (the term 'ultra-low-power Bluetooth' is still under discussion and may be changed to 'ultra-low-energy (ULE) Bluetooth'). The idea

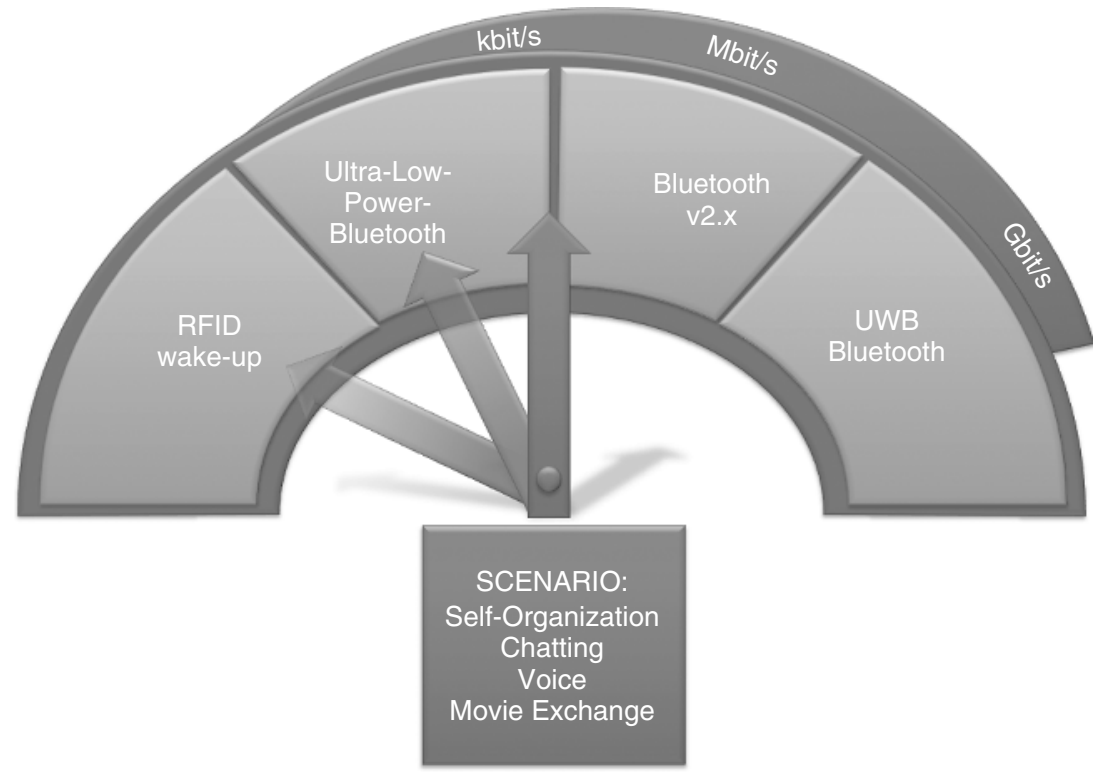

Figure 1.4 Possible Bluetooth evolution 
is to find a solution to bundle peripherals such as watches with mobile phones. Watches have only a small energy budget, and the information they want to exchange is very small. WiBree was introduced for such cases, and was taken over by Bluetooth SIG as ULP Bluetooth. A further step in this direction is the use of near-field communication to bundle Bluetooth devices. This is already being implemented in Bluetooth version 2.1. In this case, the bundling of Bluetooth devices is realized by bringing the devices very close together (a maximum distance of $2 \mathrm{~cm}$ ). This kind of bundling is very quick, but needs more user interaction. In the future, this will be realized even by RFIDs. As the current implementation in Bluetooth version 2.1 is based on UHF and therefore on active elements, RFID could be done in a total passive way. The passive way would have many advantages. Besides being very cheap, RFID technology could offer improvements in the area of energy savings.

\subsection{Mobile Architectures}

Here, the different architectures will be described, namely cellular networks, point-to-point networks, meshed networks, and cooperative networks. We will focus mainly on networks built by mobile devices, but the most prominent network candidates will also be introduced briefly.

\subsubsection{Cellular Networks}

In Figure 1.5 a cellular system is shown. These networks cover a larger area through multiple base stations having a fixed frequency plan assigned to avoid interference. Each mobile device will be connected only to one base station at a time, with potential handovers to other base stations if the mobile device starts to move. Being connected to a base station, the mobile device can connect to other mobile or fixed devices, using the base station as relay. Furthermore, the core network can also offer services that the mobile device can use. The core network differs in its implementation, be it a $2 \mathrm{G}$ or a $3 \mathrm{G}$ system. As this information will not be needed in this book, we refer the reader to other books in this area $[2,3]$.

\subsubsection{Short-range Point-to-Point Networks}

Point-to-point communication was the first form of wireless communication demonstrated by Marconi's experiments in 1895. As explained earlier, point-to-point radio communication, as shown in Figure 1.6, has some limitations in range for a fixed power margin. One example of point-to-point communication is the walkie-talkie, a means of half-duplex voice communication between users in close proximity. Nowadays, mobile devices are also able to exchange information and pure data in a point-to-point fashion, but such actions are always user driven (e.g. exchange of business cards, exchange of mp3 songs, etc.). This can be realized by in-built technologies such as infrared (IRDA), Bluetooth, ZigBee, or IEEE $802.11 \mathrm{~b} / \mathrm{g}$. Most consumers are using Bluetooth because it does not require direct line of sight, unlike IRDA, it can be found on most mobile devices, in contrast to ZigBee, and it is easy to set up, in contrast to IEEE802.11.

However, mobile peer-to-peer communication has much more potential than just point to point, and different possibilities will be presented below. Peer-to-peer technology is 


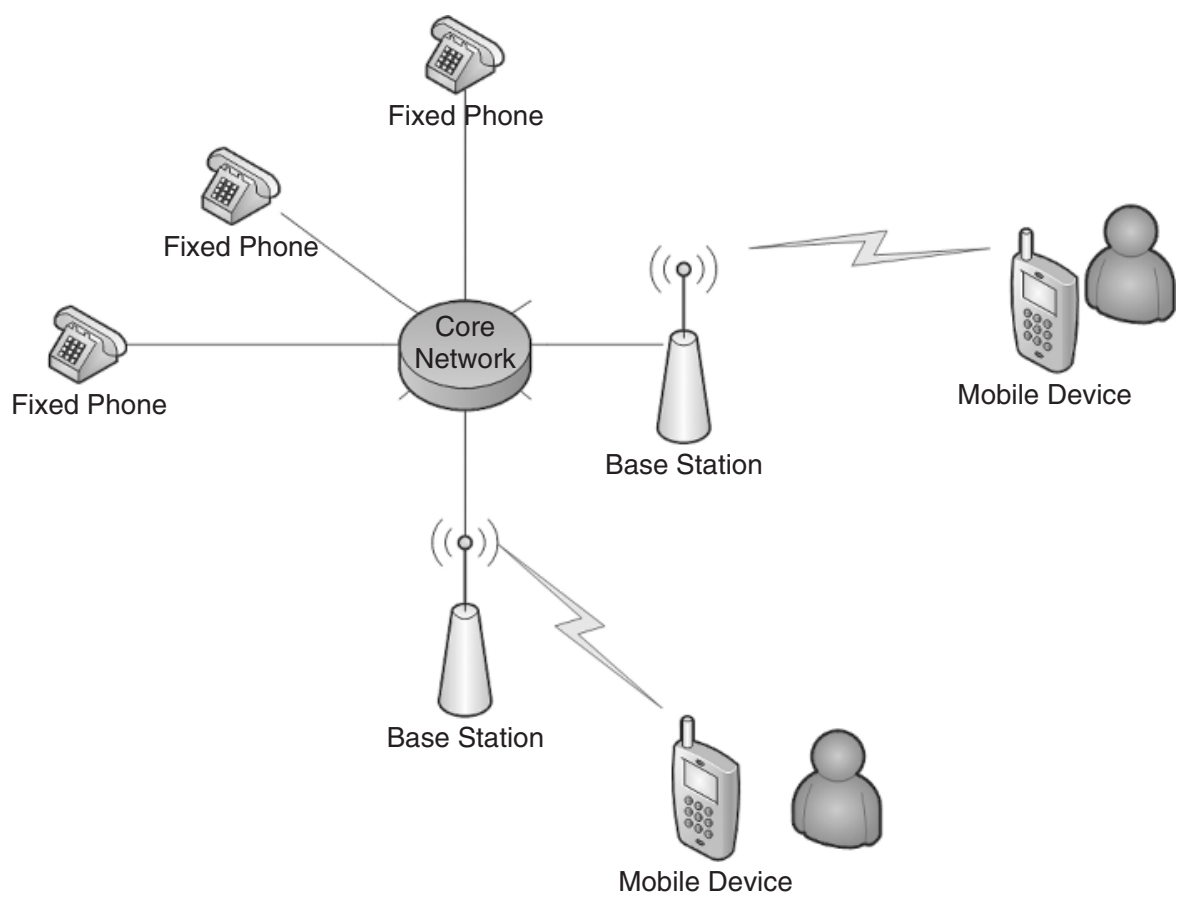

Figure 1.5 Fixed network with wireless extension

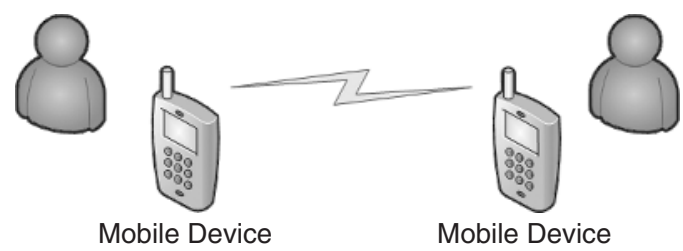

Figure 1.6 Point-to-point communication between two mobile devices

already gaining a lot of ground in fixed communication networks, and will probably also spill over to the wireless world, as laid out in this book, which advocates mobile peer to peer $(\mathrm{P} 2 \mathrm{P})$.

\subsubsection{Meshed Networks}

Meshed networks are an extension of point-to-point communication. In meshed networks, information is not always sent directly from the origin to the destination but forwarded by intermediate nodes (other mobile devices). This could be for several reasons. A first reason might be that the origin and the destination cannot communicate directly with each other. In such a case an information packet will be forwarded by the other mobile devices until it reaches the destination, as shown in Figure 1.7. Such a case is also referred 


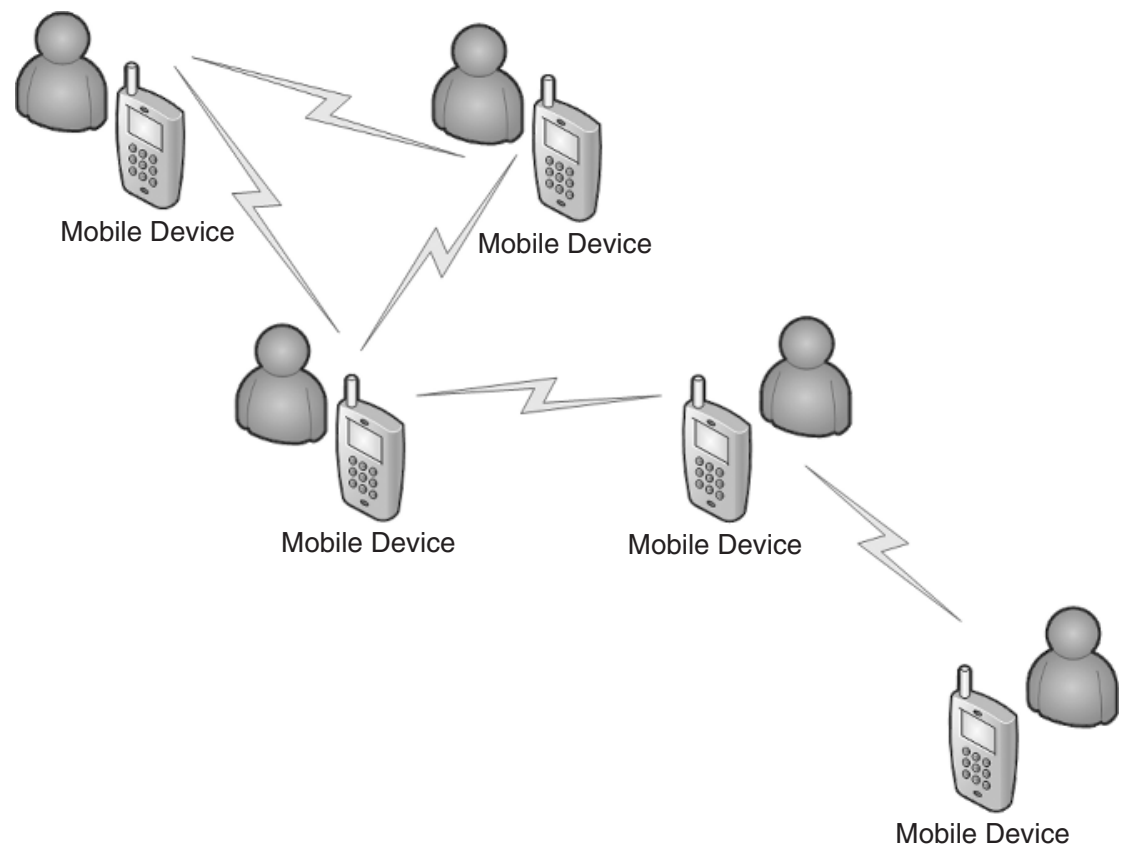

Figure 1.7 Mesh networking with four mobile devices

to as multihop. A second reason might be that, from a network perspective and maybe even from a mobile device perspective, it is more bandwidth and energy efficient to take intermediate hops instead of one hop. Whether this is true or not depends on the underlying wireless technology that is used for multihopping and the scenario. However, if we take one simple example of IEEE802.11g with rate adaptation, a single hop would use $6 \mathrm{Mbit} / \mathrm{s}$ for the transmission owing to the long distance between originator and destination. In the case of multihop, each hopping distance would be shorter and higher data rates could be used for each hop. Assuming $54 \mathrm{Mbit} / \mathrm{s}$ for every short hop, multihopping would be beneficial if less than nine hops were needed to reach the destination. This brief example demonstrates the complexity for any general architecture. Furthermore, whenever multihop is used, routing schemes are needed.

\subsubsection{Cooperative Networks}

A hybrid form of meshed networking and the cellular concept is the cooperative wireless network [4]. The main idea is that mobile devices within each other's proximity use their short-range technology to communicate directly with each other (as with meshed networks). In addition to this, each mobile device is connected to the overlay cellular or centralized network, as shown in Figure 1.8.

Using short-range technology, the mobile devices span a so-called wireless grid. The idea behind the wireless grid is that a single mobile device does not need to carry the full set of functionalities to retrieve the best possible service. Such services can be realized because the wireless grids accumulate the data rates of the participating mobile devices. 


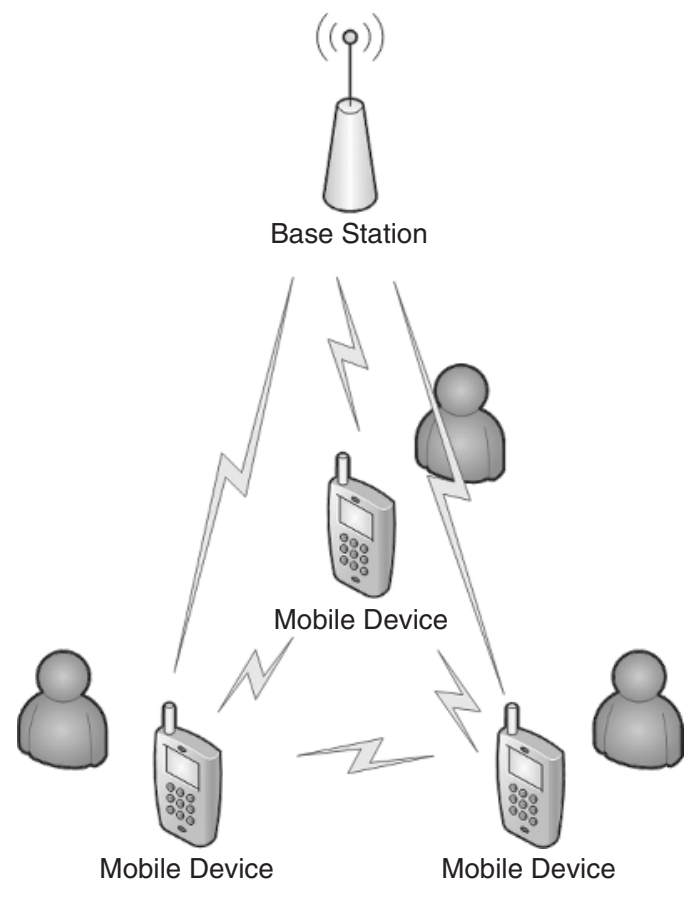

Figure 1.8 Cooperative wireless networking with three mobile devices

The alternative would be a stand-alone mobile device with high data support. However, such high data rates do not come for free. The cellular communication technology will consume more energy, which in turn will drain the battery and may lead to a heating problem of the device. The cooperating devices will only retrieve partial information from the cellular network and therefore will not drain the battery too much. The missing information will then be exchanged over the short-range links among the cooperating devices. The energy consumption will still be lower, as the energy per bit ratio on the short-range communication will be much better than on the cellular link. The cooperative concept for wireless networking was described in detail in references [4] and [5].

\subsection{Mobile Scenarios and Business Cases}

\subsubsection{Social Mobile Networks}

Social mobile networks are inspired by the established social networks found on the Internet. Social networks try to connect people who are known or unknown to each other with different goals. Social networks such as LinkedIn focus more on business-oriented people trying to extend their network for business reasons such as those given in Chapter 2 . Facebook, on the other hand, focuses more on friendship-oriented networks. Finding old friends or creating new relationships is the most important part of it. Of course, these social networks can be used on any mobile devices with a web browser. However, social mobile networks are more than just a wireless extension. 
Social mobile networks connect people who are within each other's proximity. The short-range technology is used to discover other mobile phones in the range. First solutions are on their way, such as aka-aki [6], which enriches a social network such as Facebook with collected information by short-range technology. Users can look at different profiles on the web and add connections they have made in their mobile life. The connections are collected by a mobile application running on mobile phones. The application uses Bluetooth technology to identify known or new Bluetooth contacts. Mobile users can use the application to get in contact right away or to connect up later on the web.

A similar approach is the spider application by Aalborg University [7]. As shown in Figure 1.9, the mobile user can look at a virtual world where his/her own character is moving around. As soon as Bluetooth detects other mobile phones in its proximity that are also running the spider application, more characters fill the room. Each mobile user can now steer his/her own character close to other characters and start actions such as chatting, exchanging profiles, or just looking at the real photo image. The spider approach is based on a concept developed some years ago at Aalborg University called SMARTEX [8]. The idea behind SMARTEX is to exchange digital content among mobile phones, introducing a new concept of digital ownership [9]. Again, Bluetooth technology is used here to form the mobile peer-to-peer network.

\subsubsection{Cooperative Wireless Networks}

Cooperative wireless networks are being realized at the moment. A very simplistic approach of cooperative access is realized by Jaikoo. The idea is that a mobile device that has flat-fee cellular access opens that connection to other users in its proximity. A mobile device would act as an access point to the neighbouring mobile devices, offering to share the cellular bandwidth with others. The question is: why should a user share

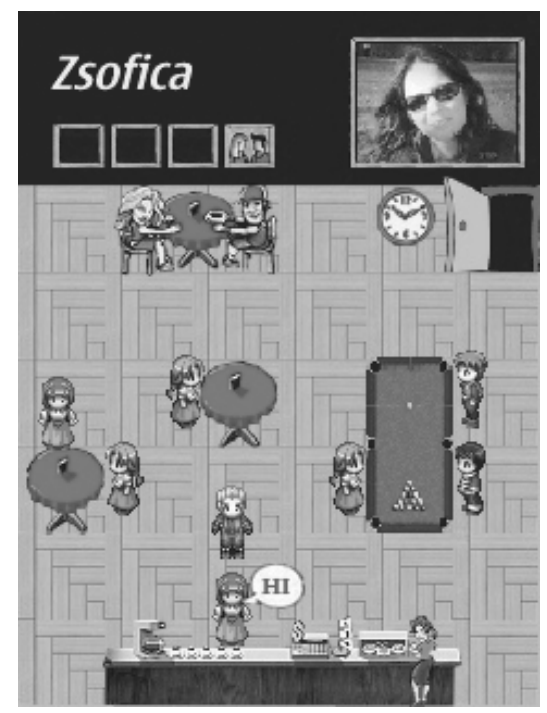

Figure 1.9 Spider screenshot 
his/her cellular connection with others? If the other users are unknown, such an act of altruistic behaviour would use up energy and cellular bandwidth that the user of the offering device would like to use for him/herself. However, such an approach could work for devices where the users know each other, for example colleagues working for the same company, friends, family members, etc. The main problem with this approach is that one user carries the entire burden while the others exploit his/her kindness.

Therefore, real cooperative wireless access has been introduced by Aalborg University. Cooperation can be realized by unknown users following the two main rules of cooperation, namely reciprocity and the detection of cheats. The idea is that multiple users will share their cellular access given a need for a cooperative cluster. Here, only two examples will be given.

In cooperative web browsing [10], the activity factor for web browsing is exploited. As the time for reading is 4 times longer than the download phase, the cellular air interface is not used most of the time. However, when it is used, the capacity of the cellular link is not fast enough. The main idea now is to bundle multiple cellular air interfaces together. Whenever a mobile device wants to download a web page, it will contact those devices that are inactive at that moment (the user is reading) to download the content of the web page in a cooperative manner. By this kind of cooperation, the download time will be reduced significantly and the cooperation will be strengthened, as all cooperating devices will be able to gain without an individual device being exploited.

The second example - the cooperative file download - will be explained in detail later in this book with code examples. If mobile users are interested in the same content, such as movies or music, they can download the content in a cooperative manner, i.e. each mobile device can download partial information of the overall information file over the cellular air interface, which can then be exchanged among the devices using short-range technology. In Chapter 7 we will explain the implementation of this idea in full.

The two business cases, cooperative wireless networking and social mobile networking, are closely related. As soon as users start to cluster for whatever reason, these two approaches can be applied. Perhaps users will start to use social mobile networks to find friends in close proximity. Once those friends are found, the mobile devices can also use this clustering to form a mobile cooperative cluster. This cluster could then offer better performance to all connected devices.

\section{References}

[1] Fitzek, F.H.P. and Reichert, F. (eds), 'Mobile Phone Programming and its Application to Wireless Networking', No. 10.1007/978-1-4020-5969-8, ISBN 978-1-4020-5968-1, Springer, Dordrecht, The Netherlands, June 2007.

[2] Eberspächer, J., Vögel, H.-J., and Bettstetter, C., 'GSM Switching, Services, and Protocols', John Wiley \& Sons, Ltd, Chichester, UK.

[3] Kaaranen, H., Ahtiainen, A., Laitinen, L., Naghian, S., and Niemi, V., 'UMTS Networks: Architecture, Mobility and Services', John Wiley \& Sons, Ltd, Chichester, UK.

[4] Fitzek, F.H.P. and Katz, M. (eds), 'Cooperation in Wireless Networks: Principles and Applications - Real Egoistic Behavior is to Cooperate!', ISBN 1-4020-4710-X, Springer, Berlin-Heidelberg-New York, April 2006.

[5] Fitzek, F.H.P. and Katz, M. (eds), 'Cognitive Wireless Networks: Concepts, Methodologies and Visions Inspiring the Age of Enlightenment of Wireless Communications', ISBN 978-1-4020-5978-0, Springer, Dordrecht, The Netherlands, July 2007. 
[6] aka-aki, http://www.aka-aki.com

[7] Sapuppo A., 'Spider Application', Aalborg University, http://mobiledevices.kom.aau.dk/projects/ student_projects/spring_2007/social_network/

[8] Pedersen, M. and Fitzek, F.H.P., 'Mobile Phone Programming - SMARTEX: the SmartME Application', ISBN 978-1-4020-5968-1 11, Springer, Dordrecht, The Netherlands, 2007, pp. 271-274.

[9] Stini, M., Mauve, M., and Fitzek, F.H.P., 'Digital Ownership: from Content Consumers to Owners and Traders', IEEE Multimedia - IEEE Computer Society, 13(5), October-December 2006, 4-6.

[10] Perrucci, G.P., Fitzek, F.H.P., Boudali, A., Canovas Mateos, M., Nejsum, P., and Studstrup, S., 'Cooperative Web Browsing for Mobile Phones', Proceedings of the International Symposium on Wireless Personal Multimedia Communications (WPMC'07), Jaipur, India, December 2007. 